Provided for non-commercial research and education use. Not for reproduction, distribution or commercial use.

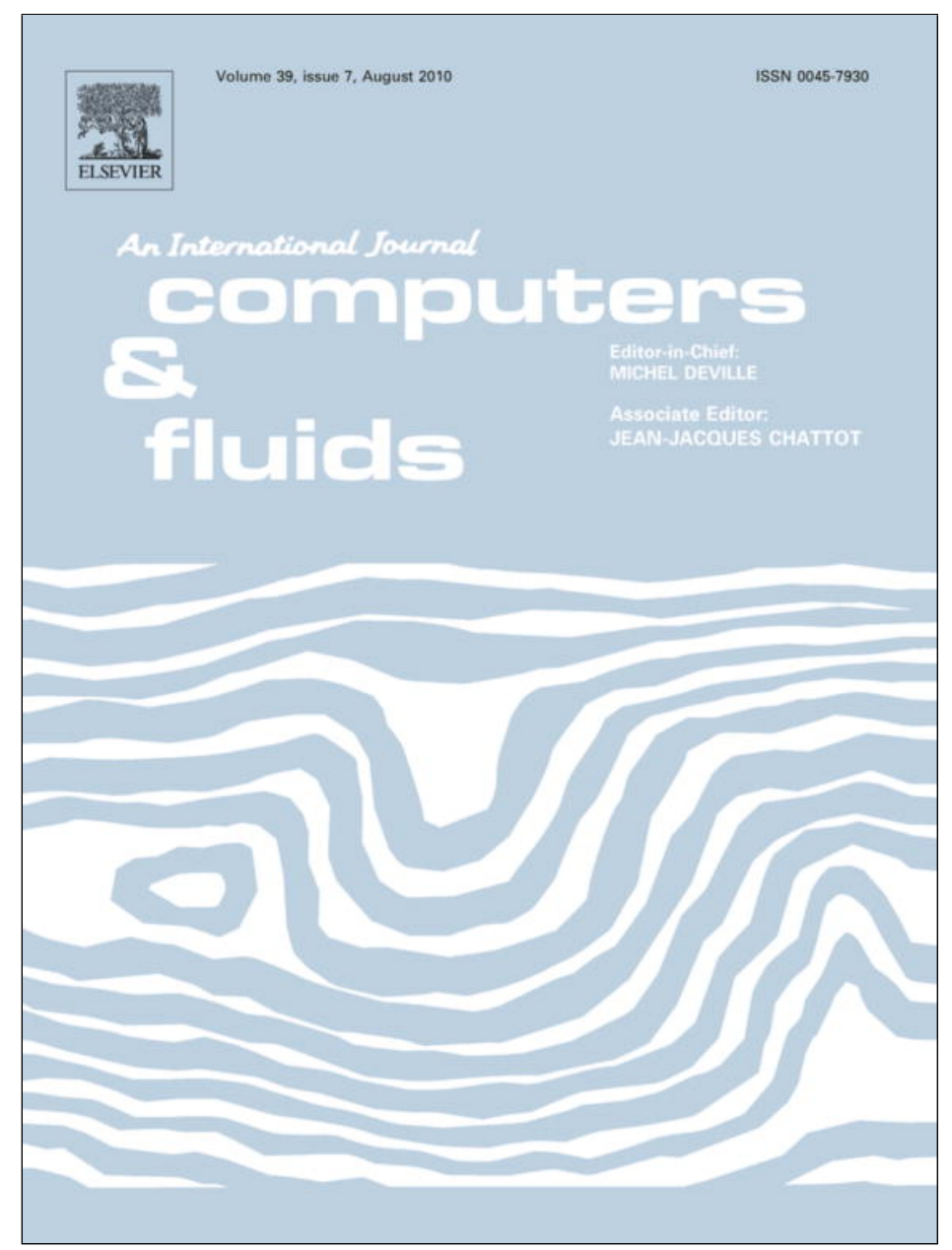

This article appeared in a journal published by Elsevier. The attached copy is furnished to the author for internal non-commercial research and education use, including for instruction at the authors institution and sharing with colleagues.

Other uses, including reproduction and distribution, or selling or licensing copies, or posting to personal, institutional or third party websites are prohibited.

In most cases authors are permitted to post their version of the article (e.g. in Word or Tex form) to their personal website or institutional repository. Authors requiring further information regarding Elsevier's archiving and manuscript policies are encouraged to visit:

http://www.elsevier.com/copyright 


\title{
Fluid-structure interaction study of the start-up of a rocket engine nozzle
}

\author{
Luciano Garelli *, Rodrigo R. Paz, Mario A. Storti \\ Centro Internacional de Métodos Computacionales en Ingeniería (CIMEC), Instituto de Desarrollo Tecnológico para la Industria Química (INTEC), \\ Consejo Nacional de Investigaciones Cientificas y Tecnológicas (CONICET), Universidad Nacional del Litoral (UNL), Güemes 3450, (S3000GLN) Santa Fe, Argentina
}

\section{A R T I C L E I N F O}

\section{Article history:}

Received 15 May 2009

Received in revised form 4 February 2010

Accepted 9 March 2010

Available online 15 March 2010

\section{Keywords:}

Fluid/structure interaction

Rocket nozzle

Aeroelasticity

Strong/weak coupling

\begin{abstract}
A B S T R A C T
The aim of this paper is to analyze the aeroelastic processes developed during the starting phase of a rocket engine via a coupling fluid/structure code. This analysis gives a better understanding of the behavior of the structure as the shock waves propagate inside the engine nozzle. The gasdynamics Euler equations are solved for the fluid and constitutive linear elastic solid assuming large displacements and rotations with no material damping is adopted for the structure. The coupling of each subproblem is carried out with a Gauß-Seidel algorithm over the fluid and structure states. For the fluid problem an ALE (Arbitrary Lagrangian-Eulerian) formulation is used. It allows us to define a reference system following the moving boundaries while the structure is deformed. The code is validated with a study of the flutter phenomena that may occur when a supersonic compressible fluid flows over a flat solid plate. Regarding the rocket engine ignition problem, a modal analysis of the structure is performed in order to analyze the eigenfrequency shifts when considering the coupling with the fluid flow.
\end{abstract}

(c) 2010 Elsevier Ltd. All rights reserved.

\section{Introduction}

Aeroelastic problems have been studied from a theoretical point of view for years, solving simplified models with few degrees of freedom [1]. Nowadays, with the development of multiphysics codes, more complex problems can be solved getting a better understanding of involved phenomena. One of these complex problems is the ignition of a rocket engine. During this stage, a sudden increase in pressure is produced in the combustion chamber, which results in compression waves that propagates through the divergent section producing deformations in the nozzle and changing the original contour. This affects directly the flow pattern and may give nonsymmetrical loads which excite the structure. The side-load phenomena has been studied by several researchers [2-5] and is caused by a non-symmetrical pressure distribution, which has a potential origin in the aeroelastic coupling. So, the key idea followed here is to include the coupling phenomena when studying the dynamic behavior and the deformation of structure during the start-up on the rocket via a transient three dimensional analysis. In addition and as is well known the effect of a dynamic load on a structure not only depends on the magnitude but also its frequency and the natural frequencies of the structure. Therefore it is of crucial interest

\footnotetext{
* Correspondence to: Luciano Garelli, Centro Internacional de Métodos Computacionales en Ingeniería (CIMEC), INTEC (CONICET-UNL), Güemes 3450, (S3000GLN) Santa Fe, Argentina.

E-mail addresses: lgarelli@intec.unl.edu.ar (L. Garelli), rodrigop@intec.unl.edu.ar (R.R. Paz), mstorti@intec.unl.edu.ar (M.A. Storti).

URL: http://www.cimec.org.ar (L. Garelli).
}

to know how the two principal natural frequencies of the structures are affected by the presence of a fluid at a high Mach number.

Large scale and complex fluid and structural problems are involved in the analysis. The simultaneous solution of the fluid and structure equations using a monolithic scheme may be mathematically unmanageable or its implementation can be a laborious task. Furthermore, the monolithic coupled formulation would change significantly if different fluid and/or structure models were considered (see Refs. [6,7]).

An efficient alternative is to solve each subproblem in a partitioned procedure using a staggered fluid/structure coupling algorithm (see Refs. [8,9]) where time and space discretization methods could be different. Such a scheme is in favor of the use of different specialized codes on each sub-area. There exist various procedures to couple the fluid and structure solvers: the coupling conditions and the moving interface can be treated in a fully explicit, implicit or in a mixed explicit/implicit manner. This approach allows a smooth transition between a weak coupling, this is when no iterations between the fluid and the structure are made and the strong coupling, this is when a number iterations between the fluid and the structure are made.

The rocket engine ignition problem is also interesting from the computational point of view as a paradigm of multiphysics code implementation that reuses preexistent fluid and solid solvers. The partitioned algorithm is implemented in the PETSc-FEM code [10] which is a parallel multiphysics finite element program based on MPI (the Message Passing Interface) and PETSc (the Portable Extensible Toolkit for Scientific Computations) library. Three instances of the PETSc-FEM code simulate each subproblem (i.e., 
fluid dynamics, mesh movement and structure dynamics problems) and communicate interface forces and displacements via standard C FIFO files or 'pipes'. The key point in the implementation of this partitioned scheme is the data exchange and synchronization between the parallel processes. These tasks are made in a small external $\mathrm{C}++$ routine.

\section{Partitioned algorithm via fixed point iteration}

In this section the temporal algorithm that performs the coupling between the structure and the fluid codes is sketched (see Ref. [11] for a detailed description). It is a fixed point iteration scheme over the variables of both fluid and structure systems. Inside of the time step loop the algorithm is equipped with an inner loop called 'stage', so if the 'stage loop' converges, then a 'strongly coupled' algorithm is obtained. Hereafter, this algorithm is called 'staggered algorithm' (see Refs. $[8,12]$ ).

The basic scheme considered in this work proceeds as follows:

(i) Transferring the motion of the wet boundary of the solid to the fluid problem.

(ii) Updating the position of the fluid boundary and the bulk fluid mesh accordingly.

(iii) Advancing the fluid system and compute new pressures.

(iv) Converting the new fluid pressure (and stress field) into a structural load.

(v) Advancing the structural system under the flow loads.

In this algorithm three codes: Computational Fluid Dynamics (CFD), Computational Structure Dynamics (CSD) and Computational Mesh Dynamics (CMD) are running simultaneously. For simplicity, the basic algorithm can be thought as if there were no 'concurrence' between the codes, i.e. at a given time only one of them is running. This can be controlled using 'semaphores' and this is done using MPI 'synchronization messages'. A schematic diagram is shown in Fig. 1.

At time $t_{n}$, we define $\mathbf{w}^{n}$ to be the fluid state vector $(\rho, \mathbf{v}, p), \mathbf{u}^{n}$ to be the displacement vector (structure state vector), $\dot{\mathbf{u}}^{n}$ the structure velocities and $\mathbf{X}^{n}$ the fluid mesh node positions. In this work, both fluid and structure partitions are integrated with the trapezoidal algorithm (with trapezoidal parameter $0<\alpha_{\text {trap }} \leqslant 1$ ). In each time step the fluid is first advanced using the previously computed structure state $\mathbf{u}^{n}$ and the current estimated value $\mathbf{u}_{p}^{n+1}$. In this way, a new estimation for the fluid state $\mathbf{w}^{n+1}$ is computed. Next the structure is updated using the forces of the fluid from states $\mathbf{w}^{n}$ and $\mathbf{w}^{n+1}$. The estimated state $\mathbf{u}_{p}^{n+1}$ is predicted using a second or higher order approximation, were $\alpha_{0}$ and $\alpha_{1}$ are two real constants. The prediction (1) is trivial if $\alpha_{0}=\alpha_{0}=0$, first-order time-accurate if $\alpha_{0}=1$ and second-order time-accurate if $\alpha_{0}=1$ and $\alpha_{1}=1 / 2$. In Ref. [9] there is an extended description about the use of the predictor (1) on fluid-structure interaction problems and the energy transfer between the fluid and the structure. It was proved that monolithic schemes and strongly-coupled staggered schemes conserve energy-transfer at the fluid-structure interface boundary, whereas weak-coupled algorithms introduce after a certain amount of time $t$ an artificial energy $E=\mathcal{O}\left(\Delta t^{p}\right)$, where $p$ is the order of the prediction.

$\mathbf{u}_{p}^{(n+1)}=\mathbf{u}^{n}+\alpha_{0} \Delta t \dot{\mathbf{u}}^{n}+\alpha_{1} \Delta t\left(\dot{\mathbf{u}}^{n}-\dot{\mathbf{u}}^{n-1}\right)$.

Once the coordinates of the structure are known, the coordinates of the fluid mesh nodes are computed by a 'Computational Mesh Dynamics' code, which is symbolized as:

$\mathbf{X}^{n}=\operatorname{CMD}\left(\mathbf{u}^{n}\right)$.

The movement of the mesh can be performed with a general strategy using both nodal relocation or re-meshing. In this paper only the former is adopted, keeping the topology unchanged. The relocation of mesh nodes can be done using an elastic or pseudoelastic model (see Ref. [13]) or minimizing a functional that measures the distortion of the mesh with a geometric quality indicator (see Ref. [14]) through a separate PETSc-FEM parallel process. Both schemes are available in the PETSc-FEM code.

At the beginning of each fluid stage there is a computation of skin normals and velocities. This is necessary due to the time dependent slip boundary condition for the inviscid case, implemented as a constraint (see Eq. (9)), and also when using a non-slip boundary condition, where the fluid interface has the velocity of the moving solid wall, i.e., $\left.\mathbf{v}\right|_{\Gamma}=\left.\dot{\mathbf{u}}\right|_{\Gamma}$.

\section{Description of the validation test}

Prior to focus on the aeroelastic behavior of the rocket nozzle and in order to validate the code, the flutter of a flat solid plate aligned with a gas flow at supersonic Mach number (see Fig. 2) is studied and the critical Mach number is computed. This test can be seen as a very simple representation of an isolated portion of the wall nozzle and is relevant because the onset of physical instabilities is very sensitive to the precision in the transfer of forces and displacements. A thorough description can be found in $[9,11]$ together with a deeper analysis on stability and convergence of the algorithm.

The flutter mechanism is a convergence of natural frequencies with increasing flow velocity. The flutter frequency is between the first and second natural frequencies and the mode shape shows

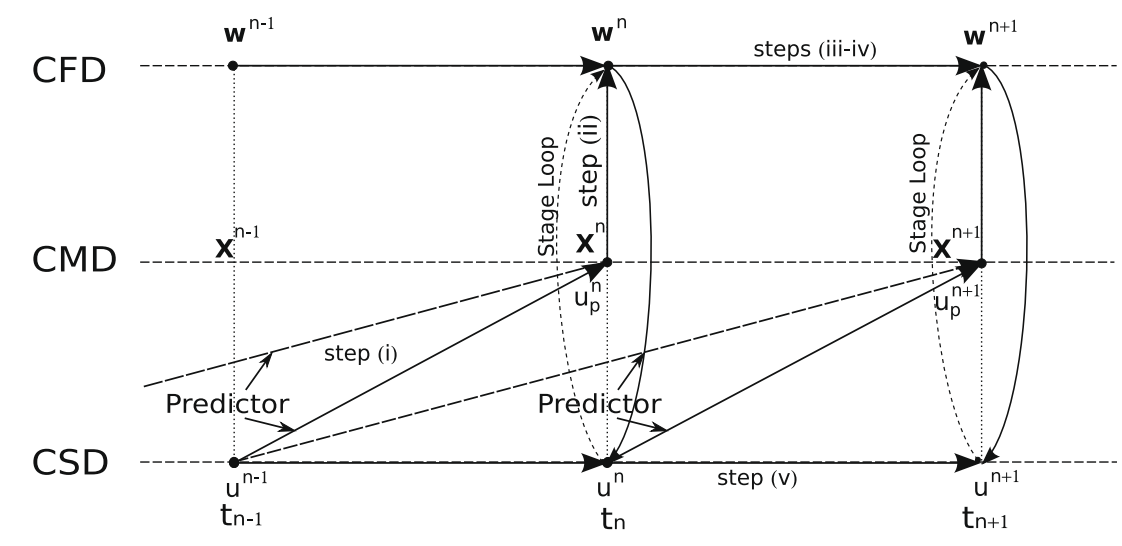

Fig. 1. Synchronous FSI partitioned scheme. 


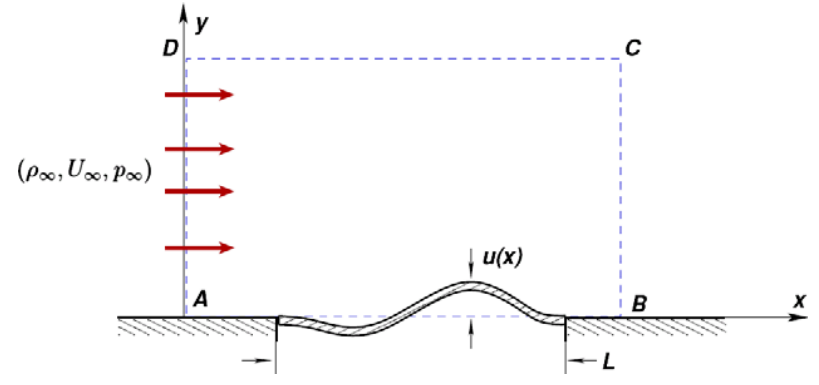

Fig. 2. Description of the test case.

a maximum on the rear edge of the plate, rather than on the front edge (see Ref. [1]).

A uniform fluid at state $\left(\rho_{\infty}, U_{\infty}, p_{\infty}\right)$ flows over an horizontal rigid wall (locate at $y=0$ ) parallel to it. In a certain region of the wall $(0 \leqslant x \leqslant L)$ the wall deforms elastically following thin plate theory, i.e.

$m \ddot{u}+D \frac{\partial^{4} u}{\partial x^{4}}=-\left(p-p_{\infty}\right)+f(x, t)$,

where $m$ is the mass of the plate per unit area in $\left(\mathrm{kg} / \mathrm{m}^{2}\right)$, $D=E d^{3} / 12\left(1-v^{2}\right)$ the bending rigidity of the plate module in $(\mathrm{N} \mathrm{m}), E$ is the Young modulus in $(\mathrm{Pa}), d$ the plate thickness in (m), $v$ the Poisson modulus, $u$ the normal deflection of the plate in $(\mathrm{m})$, defined on the region $0 \leqslant x \leqslant L$ and null outside this region, $p$ the pressure exerted by the fluid on the plate in (Pa), $f$ is an external force in $(\mathrm{N})$.

All variables and parameters are dimensionless by selecting $L / 2=1(\mathrm{~m}), \rho_{\infty}=1\left(\mathrm{~kg} / \mathrm{m}^{3}\right)$ and $c_{\infty}=\sqrt{\gamma p_{\infty} / \rho_{\infty}}=1(\mathrm{~m} / \mathrm{s})$ as reference values for length, mass and time scales.

The following parameters are used:

$\rho_{\infty}=1$,

$p_{\infty}=1 / \gamma=0.71429(\gamma=1.4)$

$U_{\infty}=M_{\infty}\left(\right.$ since $\left.c_{\infty}=1\right)$,

$D=0.031611$,

$m=36.585$

$L=2$.

The plate is clamped at both ends, i.e. $u=\frac{\partial u}{\partial x}=0$ at $x=0, L$. For the sake of simplicity the fluid occupying the region $y>0$ is inviscid. The gasdynamics Euler equations with SUPG (Streamline Upwind Petrov Galerkin) stabilization and 'anisotropic shockcapturing' method are considered (see Refs. [15,16]).

A slip condition is assumed

$\left(\mathbf{v}-\mathbf{v}_{s t r}\right) \cdot p \hat{\mathbf{n}}=0$

on the (curved) wall $y=u(x)$, where

$\mathbf{v}_{\text {str }}=(0, \dot{u})$,

$\hat{\mathbf{n}} \propto\left(-\frac{\partial u}{\partial x}, 1\right)$,

are the velocity of the plate and its unit normal. Finally, initial conditions for both the fluid and the plate are taken as

$$
\begin{aligned}
& u(x, t=0)=u_{0}(x), \\
& \dot{u}(x, t=0)=\dot{u}_{0}(x), \\
& (\rho, \mathbf{v}, p)_{\mathbf{x}, t=0}=(\rho, \mathbf{v}, p)_{0}, \quad \text { for } \mathrm{y} \geqslant u_{0}(\mathbf{x}) .
\end{aligned}
$$

Note that the free stream fluid pressure is subtracted and in the absence of any external perturbation $(f \equiv 0)$ the undisturbed flow $(\rho, \mathbf{v}, p)_{x, t} \equiv(\rho, \mathbf{v}, p)_{\infty}$ is a solution of the problem for the initial conditions

$$
\begin{aligned}
& u \equiv 0 \\
& \dot{u} \equiv 0, \\
& (\rho, \mathbf{v}, p)_{\mathbf{x}, t=0} \equiv(\rho, \mathbf{v}, p)_{\infty} .
\end{aligned}
$$

The study of the flutter instability is carried out by means of the modal analysis, assuming the 'Houbolt approximation' for the fluid (see Ref. [19]), where the pressure acting on the plate surface is a function of the plate deflection derivatives, i.e., $p-p_{\infty}=f\left(\frac{\partial u}{\partial x}, \frac{\partial u}{\partial t}\right)$. Then a Galerkin method is used and the normal displacement is expanded in a global basis. These basis functions satisfy the essential boundary conditions for the plate equation $u=\frac{\partial u}{\partial x}=0$ at $x=0, L$. Replacing the Houbolt approximation in Eq. (3), using Galerkin method and integrating by parts as needed, leads to the eigenvalue problem.

Flutter is detected whenever the real part of some eigenvalue $\lambda$ changes its sign. In order to determine the critical Mach number $M_{c r}$, the interval $1.8 \leqslant M \leqslant 3.0$ was swept with increments of 0.01 .

For this problem all the eigenvalues have negative real part for $M_{\infty}<M_{c r}=2.265$ which results in a stable system. For $M_{\infty}>M_{c r}=2.265$ there are two complex conjugate roots with positive real parts. The computed value of $M_{c r}$ is in agreement with the result given in [9] (i.e., $M_{c r}=2.23$ ). These results will be used to validate the coupling code.

\subsection{FSI code results}

The aeroelastic problem defined above was modeled with the partitioned algorithm described in Section 2 using the weak coupling scheme between fluid and structure, i.e. $n_{\text {stage }}=1$. A mesh of 12,800 quadrilateral elements for the fluid and other of 5120 for the plate were used for the spatial discretization and the CrankNicolson scheme with a variable time step per case was used, based on a Courant number of 5 for the unperturbed flow. This amounts to $\Delta t=0.02762$ for Mach 2.225 and $\Delta t=0.0272$ for Mach 2.2275.

The setting of boundary conditions at the inlet and outlet walls is based in the number of incoming and outgoing characteristics on these boundaries. Therefore, at the inlet wall all four characteristics are incoming so that, four variables $[\rho, u, v, p]$ are specified as Dirichlet conditions. At the outlet wall, all four characteristics are outgoing so that, no (Dirichlet) conditions are specified. Therefore, the problem is well-posed and no wave reflection occurs.

As the flow is supersonic only a small entry section of $1 / 8 \mathrm{~L}$ upstream the plate and $1 / 3 L$ downstream is considered. The vertical size of the computational domain was chosen as $0.8 \mathrm{~L}$. This choice for the vertical size guarantees that no reflection from the upper boundary pollutes the region of the plate.

In fluid structure interaction problems solved with ALE schemes [20], it is known that, the mesh velocity depends on the fluid-solid interface velocity, therefore, to guarantee second order in time accuracy it is necessary to use $\alpha_{0}=1$ and $\alpha_{1}=1 / 2$ for the predictor. Note that, if the Crank-Nicolson scheme is used for the time integration of both the structure and the fluid equations and the predictor is chosen with at least second order precision, then the whole algorithm is second order, even if only one stage is performed (see Ref. [12]).

In order to find (numerically) the critical Mach number for this problem a sweep in the Mach number in the range of 1.8-3.2 was done. Results for some Mach numbers can be seen in Fig. 3. In these plots, the time evolution of displacements of twenty equispaced points along the skin plate are shown. In figures corresponding to $M=2.275$ it is clearly appreciable the divergent tendency on the plate deflection. This means that the flutter condition has been reached. The fluid density field and the structure displacement at Mach $=3.2$ (flutter region) for a given time step is shown in Fig. 4. 

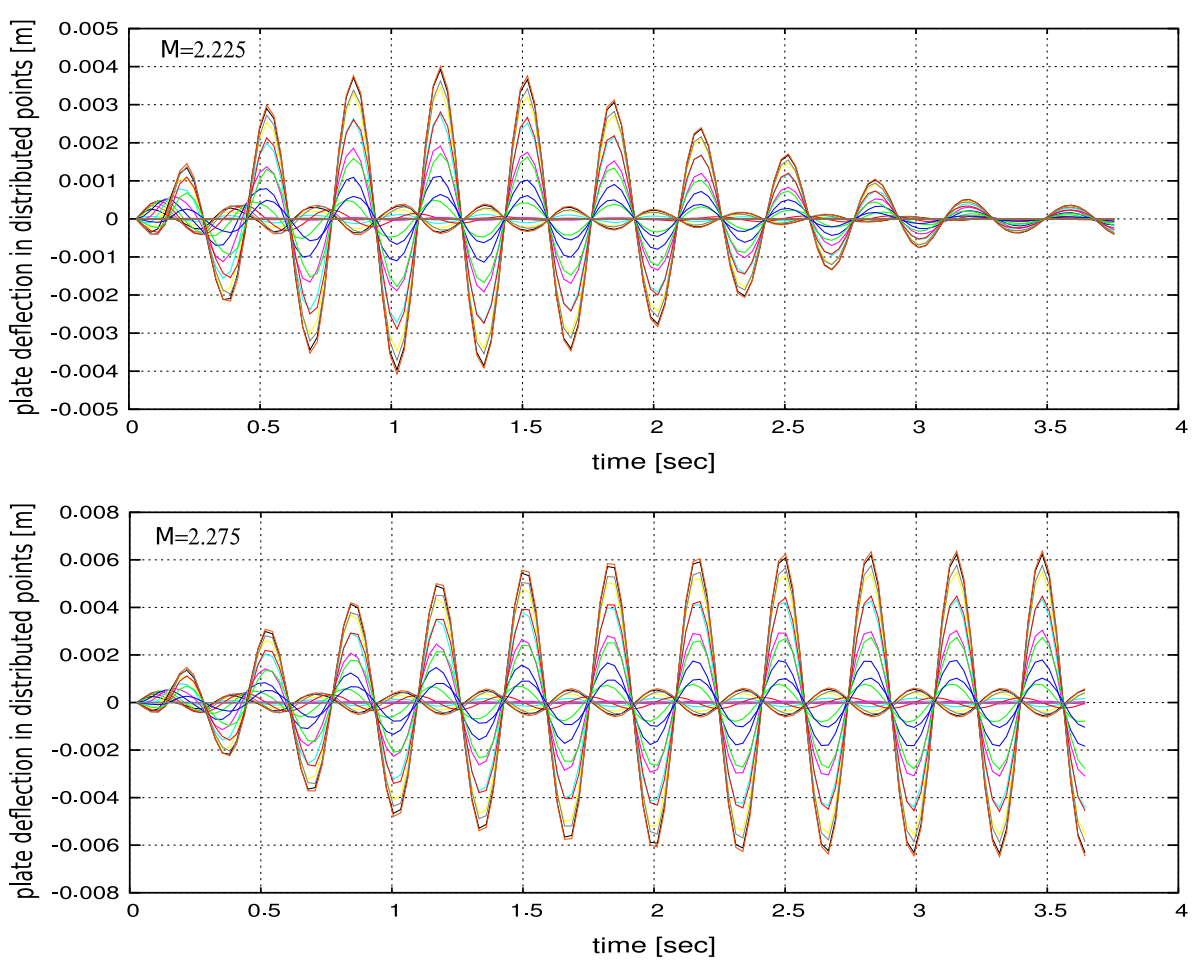

Fig. 3. Plate deflection in distributed points along plate.

\section{Rocket ignition: description of proposed analysis}

The algorithm described and validated in Sections 2 and 3 will be used to obtain the deformation in the nozzle of a rocket engine during the ignition. This problem has been under study by many researchers over the years, carrying both numerical [21-24] and experimental $[25,26]$ analysis.

Nozzles with high area ratio are used in the main space launchers (Space Shuttle Main Engine, Ariane 5). These engines must work in conditions ranging from sea level to orbital altitude but an efficient operation is reached only at high altitude. The nozzles contour is often designed according to the theory proposed by Rao [27] that results in Thrust Optimized Parabolic or Parabolic Bell (TOP) nozzle, which has some advantages compared to the traditional conical shapes. These advantages are the smaller length, lower weight, as well as the reduction in energy losses in the expansion of gases [28-31].

During the start-up phase the structure is deformed due to the advance of a shock wave that is highly detrimental to the integrity and service life cycle of the rocket engine. Many problems have

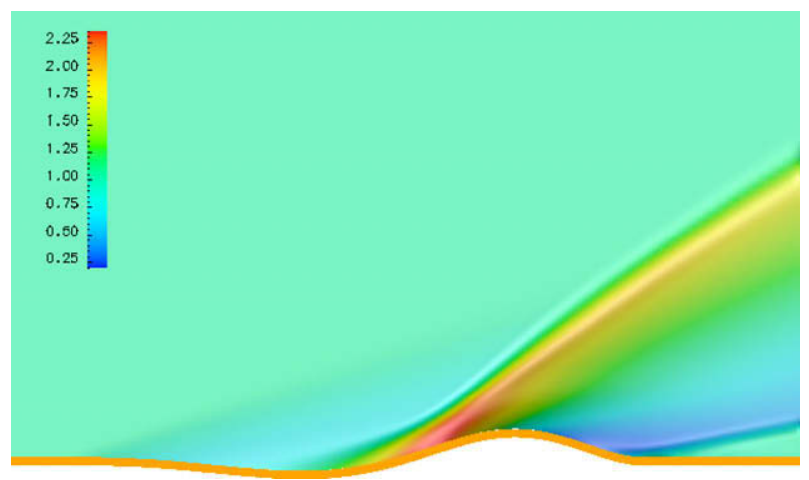

Fig. 4. Fluid and structure fields at $M=3.2$. Colormap = fluid density, time $=1.68 \mathrm{~s}$. been encountered in the Space Shuttle Main Engine, European Vulcain (Ariane) and in the Japanese LE-7, all these were related to the ignition stage and side loads phenomena.

The nozzle under study has a bell-shape geometry which is generated by rotating a contour line around the $x$ axis. In this way the 3D geometry is obtained (see Fig. 5).

The most relevant geometrical data are detailed below:

- Overall length: $l=1810(\mathrm{~mm})$.

- Throat diameter: $D_{t}=304(\mathrm{~mm})$.

- Exit diameter: $D_{e}=1396(\mathrm{~mm})$.

- Area ratio: $\epsilon=21.1$.

\subsection{Numerical model}

Starting from the three-dimensional model two independent meshes are generated, one for the fluid domain discretization and the other for the structure domain discretization. A mesh with 334,700 tetrahedral elements is generated for the fluid with a linear interpolation of the variables. The structural mesh is composed of 59,600 wedge (triangular base prismatic) elements. Detailed view of grid zones of both meshes is shown in Fig. 6 .

In FSI problems there is an information transfer in the fluidstructure interface. Using conforming meshes (node to node coincident) on the interface, the transmission is direct and does not need an algorithm to do a surface tracking, state interpolation and load projection, but the major drawback of this method is that refinement in the structure mesh will cause an increase in the fluid mesh and therefore in the overall problem size.

The structural problem is solved using a PETSc-FEM module, which is based on the theory of constitutive linear elastic material, geometrically nonlinear and no material damping. The gasdynamics Euler equations are solved and SUPG stabilization is used together with the shock-capturing method which has been proposed by Tezduyar and Senga [15]. Using the Euler equations the CPU and memory costs can be significantly reduced compara- 

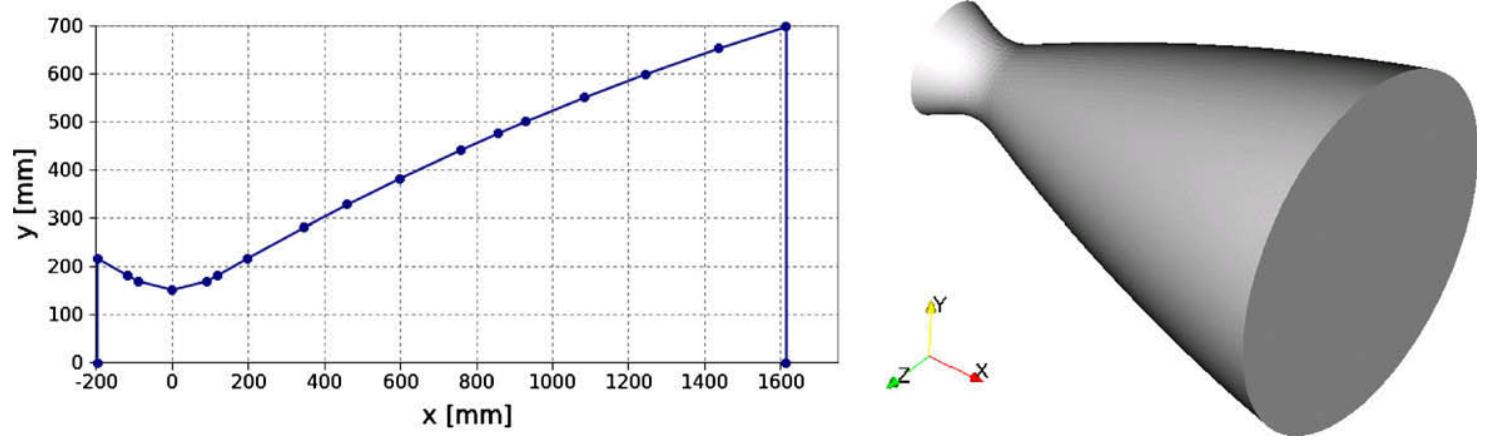

Fig. 5. Contour line and 3D model.
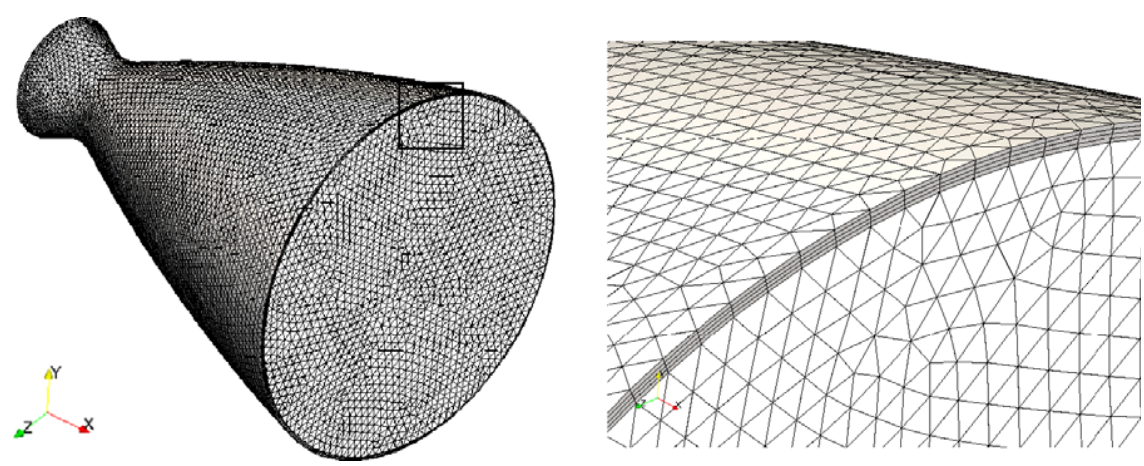

Fig. 6. Spatial discretization for the fluid and for the structure

tively to the viscous case. Furthermore, from previous works $[17,18]$ can be concluded that this equations correctly predict the main flows feature.

The data exchange and synchronization between the three parallel processes are carried out with an external $\mathrm{C}++$ routine, jointly with the pressure integration over the interface to get the force acting on the structure.

The strategy adopted in this work to solve an aeroelastic problem would be useful when analyzing the stability of nozzles, and it will be considered in a future work. In the early 1990s simplified techniques for analyzing the stability were proposed by Pekkari $[2,3]$, where the parietal pressure due to the wall deformation is giving by an analytical expression. A recent work carried out by Östlund [4] made an improvement in the technique. But these methods solve the aeroelastic problem in a decoupled fashion.

In order to solve the aeroelastic problem the material properties for the nozzle and fluid are summarized in Tables 1 and 2. In this work the nozzle is modeled with an homogeneous material, but more complex structural models can be similarly used.

\subsection{Boundary and initial conditions for the nozzle ignition problem}

The FSI problem requires initial and boundary conditions for both, the structural and the fluid problem, separately. The nozzle is clamped (all displacements null) at the junction with the combustion chamber and the rest is left free. In the fluid flow problem a slip condition is applied to the wall of the nozzle, which is mathematically represented by the following equation:

Table 1

Solid properties.

\begin{tabular}{llll}
\hline Young's modulus & Poisson's coefficient & Density & Thickness \\
\hline $2.07 \times 10^{10}\left(\mathrm{~N} / \mathrm{m}^{2}\right)$ & 0.28 & $8400\left(\mathrm{~kg} / \mathrm{m}^{3}\right)$ & $0.015(\mathrm{~m})$ \\
\hline
\end{tabular}

Table 2

Fluid properties.

\begin{tabular}{lllll}
\hline$R$ & $\gamma$ & $\rho_{\infty}$ & $T_{\infty}$ & $p_{\infty}$ \\
\hline $287(\mathrm{~J} / \mathrm{kg} \mathrm{K})$ & 1.40 & $1.225\left(\mathrm{~kg} / \mathrm{m}^{3}\right)$ & $288(\mathrm{~K})$ & $10,1253(\mathrm{~Pa})$ \\
\hline
\end{tabular}

$\left(\mathbf{v}-\mathbf{v}_{s t r}\right) \cdot \hat{\mathbf{n}}=(\mathbf{v}-\dot{\mathbf{u}}) \cdot \hat{\mathbf{n}}=0$

Mentioned above the slip condition must be applied dynamically because the normal to the wall and the structure velocity change during the simulation. For the fluid $\left(p_{0}, T_{0}\right)$ are imposed at the inlet. These conditions are taken from the stagnation condition of the combustion chamber $\left(p_{0}, T_{0}\right)$, and then $\rho_{0}$ is computed from the state equation (see Table 3 ). The modeling of the ignition of a rocket exhaust nozzle is challenging from several points of view. One of these points is the imposition of boundary conditions that at the outlet wall must be non-reflective. Moreover, in such case, the needed conditions at the outlet boundary change from rest (i.e., subsonic flow) to supersonic flow as a shock wave appears at the throat and propagates toward the boundary. So, the condition must be capable of handling the dynamical change of the Jacobians matrix profile. During the flow computation inside the nozzle the number of incoming/outgoing characteristics, and therefore the number of Dirichlet conditions to be imposed, will change. Having a boundary condition that can automatically adapt itself to this change is essentially useful in such a problem. In addition, the computational domain can be limited to the nozzle interior up to the exit plane, with a significant reduction in CPU time and memory use. Imposing absorbent/dynamic boundary conditions is based on the analysis of the projection

Table 3

Stagnation values used for the combustion chamber.

\begin{tabular}{lll}
\hline$p_{0}$ & $\rho_{0}$ & $T_{0}$ \\
\hline $26(\mathrm{MPa})$ & $306.25\left(\mathrm{~kg} / \mathrm{m}^{3}\right)$ & $299(\mathrm{~K})$ \\
\hline
\end{tabular}


Table 4

Characteristic dimensions.

\begin{tabular}{llll}
\hline Nozzle & Vulcain & S1 & S3 \\
\hline Area ratio $(\epsilon)$ & 45 & 20 & 18.2 \\
Nozzle length $(L)(\mathrm{mm})$ & 2065.5 & 350 & 528.2 \\
Throat diameter $\left(D_{\mathrm{t}}\right)(\mathrm{mm})$ & 262.4 & 67.08 & 67.08 \\
Nozzle exit diameter $\left(D_{\mathrm{e}}\right)(\mathrm{mm})$ & 1760.2 & 300.0 & 286.5 \\
\hline
\end{tabular}

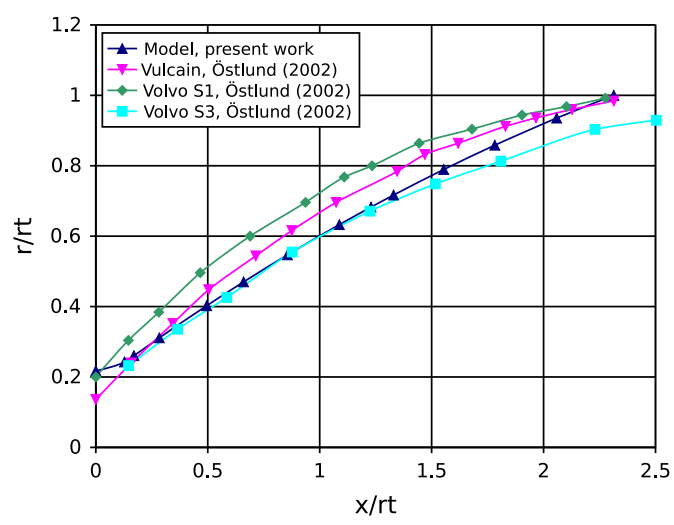

of the Jacobians of advective flux functions onto normal directions to fictitious surfaces. The advantage of the method is that it is very easy to implement and that it is based on imposing non-linear constraints via Lagrange Multipliers or Penalty Methods (see Ref. [32] for a more detailed description).

Initial conditions must be established in both domains. The following are adopted for the fluid

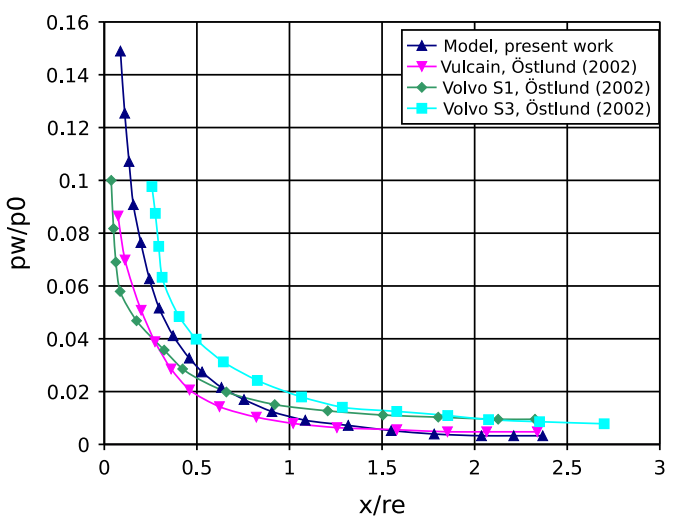

Fig. 7. Radius and wall pressure distribution.

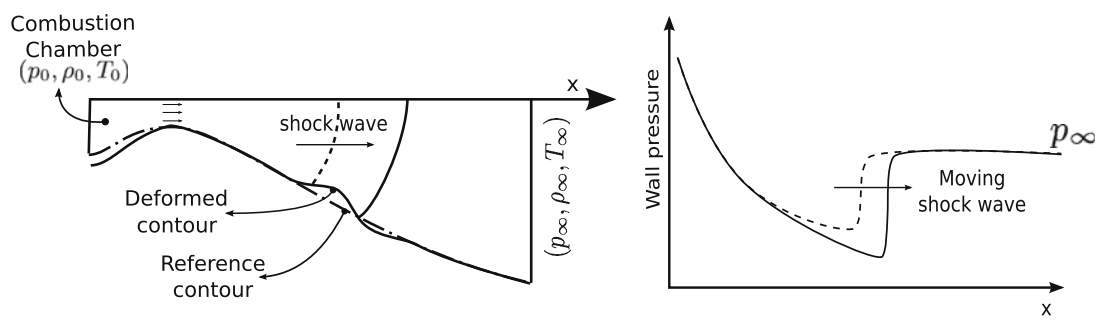

Fig. 8. Schematic deformation of the structure.
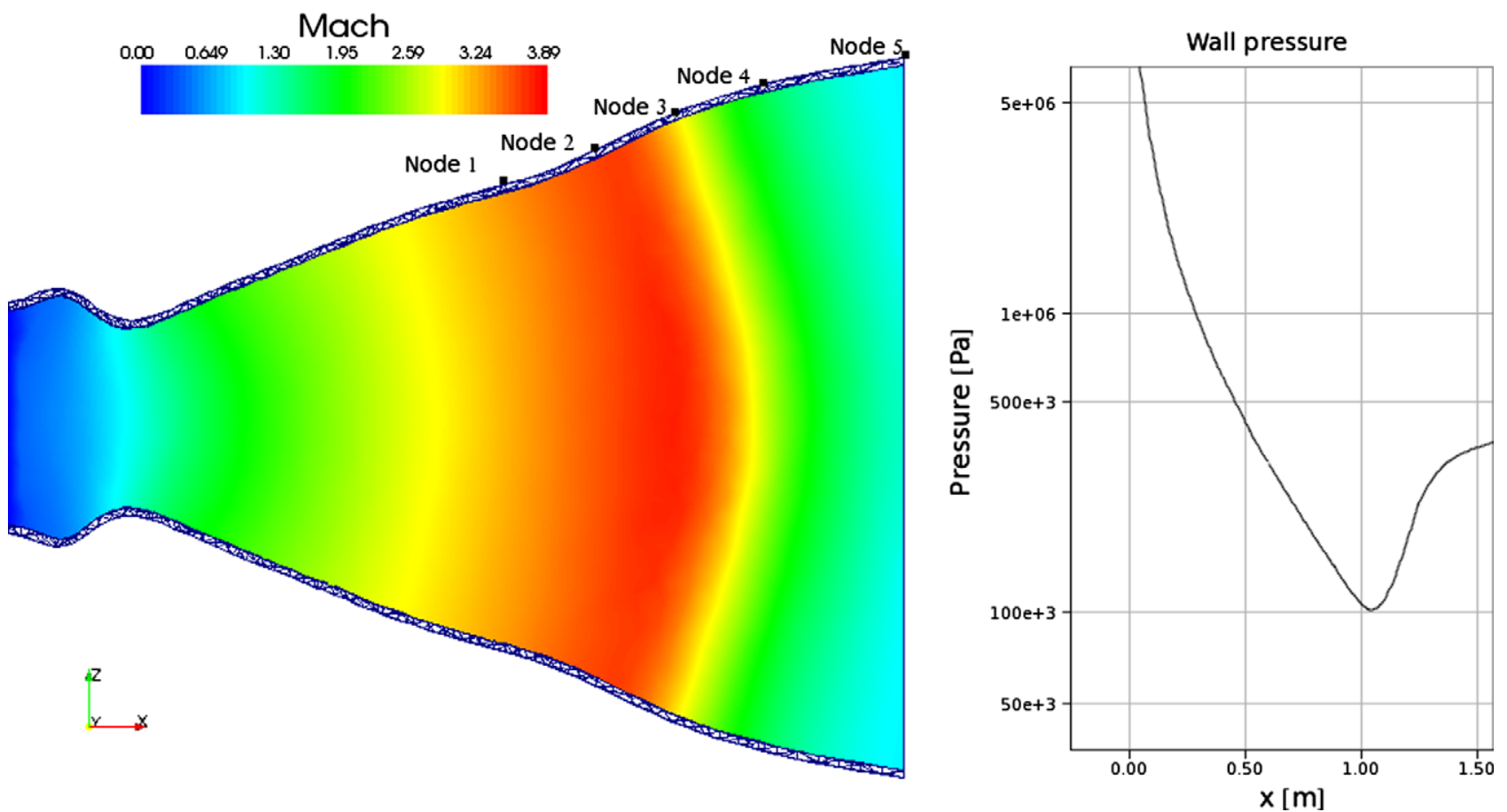

Fig. 9. Structure deformation and pressure distribution for the moving shock wave. 
$\mathbf{v}\left(\mathbf{x}, t_{0}\right)=0$

$p\left(\mathbf{x}, t_{0}\right)=p_{\infty}$

$\rho\left(\mathbf{x}, t_{0}\right)=\rho_{\infty}$,

and the next for the structure

$\mathbf{u}\left(\mathbf{x}, t_{0}\right)=0$

$\dot{\mathbf{u}}\left(\mathbf{x}, t_{0}\right)=0$.

\section{Results}

\subsection{Aeroelastic behavior of the nozzle}

The proposed numerical problem was carried out on a cluster [33] machine using 30 processors Intel ${ }^{\circledR}$ Pentium $^{\circledR}$ IV Prescott
$3 \mathrm{GHz}$ with $2 \mathrm{~Gb}$ of RAM (DDR2 $400 \mathrm{MHz}$ ), interconnected with two switch Gigabit Ethernet (1 Gbit/s), $3 \mathrm{Com}^{\circledR}$ Super Stack 3. In the simulation, 2000 time steps with a $\Delta t=2 \times 10^{-5}$ (s) are computed to obtain a fully developed flow, taking into account that the shock wave leaves the interior of the nozzle in approximately $8.8 \times 10^{-3}(\mathrm{~s})$.

Before performing the aeroelastic analysis, the nozzle used in this work is compared to the Vulcain nozzle and to the sub-scale S1 and S3 nozzles (see Table 4) through a parametric study that was carried out in [5]. That is done because the fluid flow field is determined by the shape of the nozzle and this affects the pressure distribution on the wall from which the fluid loads are computed.

In the S1 sub-scale nozzle the characteristic length for the scaling was the nozzle exit radius $\left(r_{e}\right)$ and in the S3 sub-scale nozzle was the throat radius $\left(r_{t}\right)$, thus different contours are obtained.

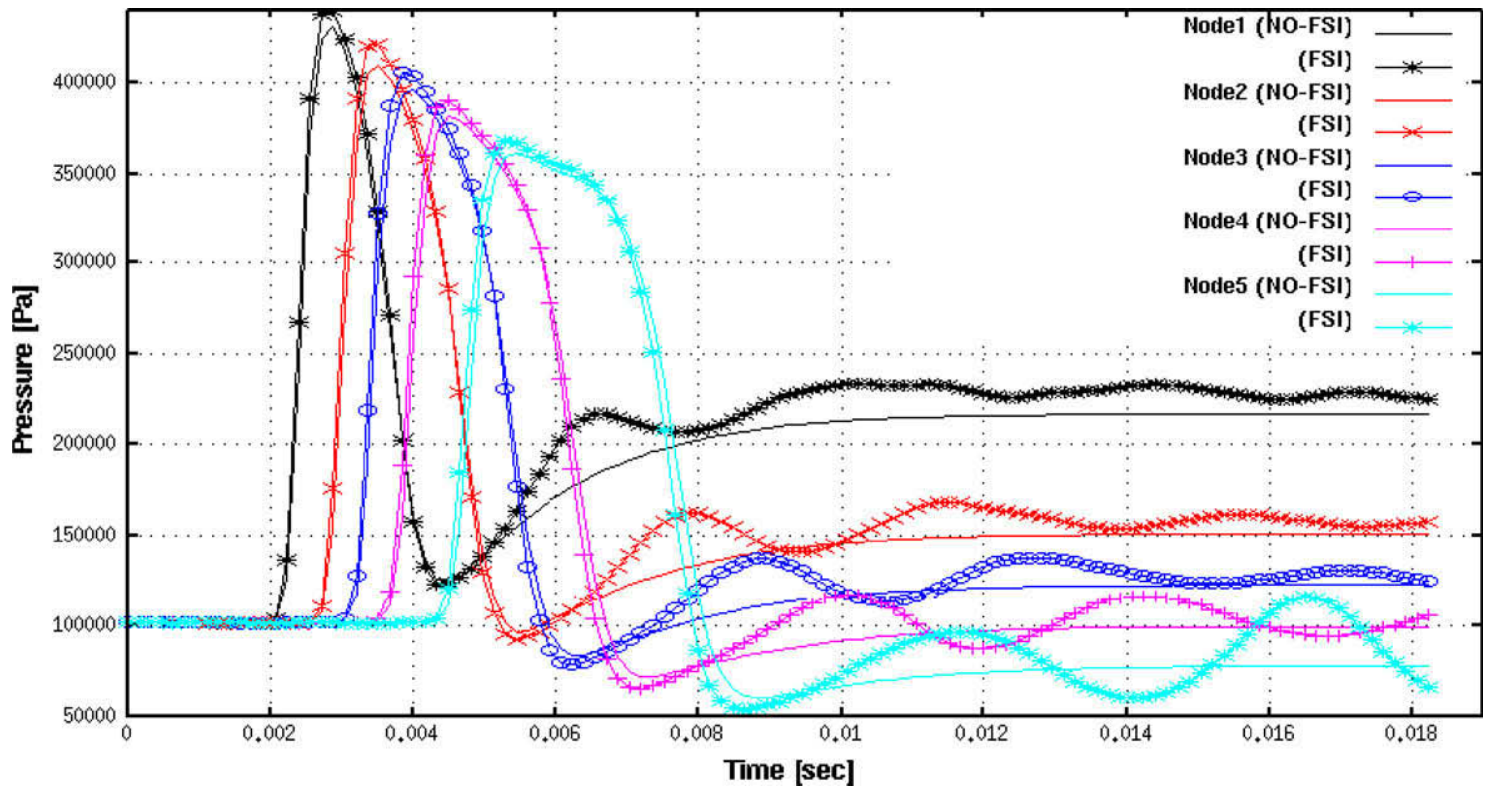

Fig. 10. Wall pressures during the start-up. Comparison between NO-FSI and FSI cases.

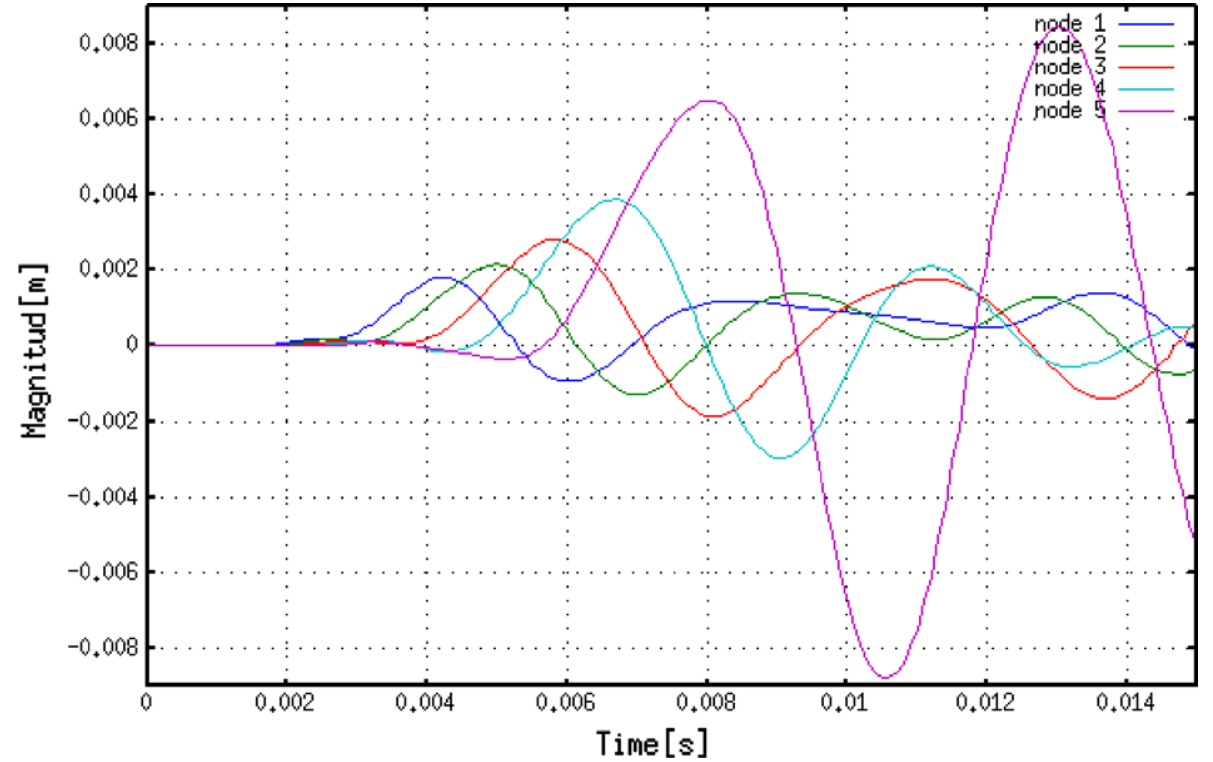

Fig. 11. Displacement of nodes 1-5. 

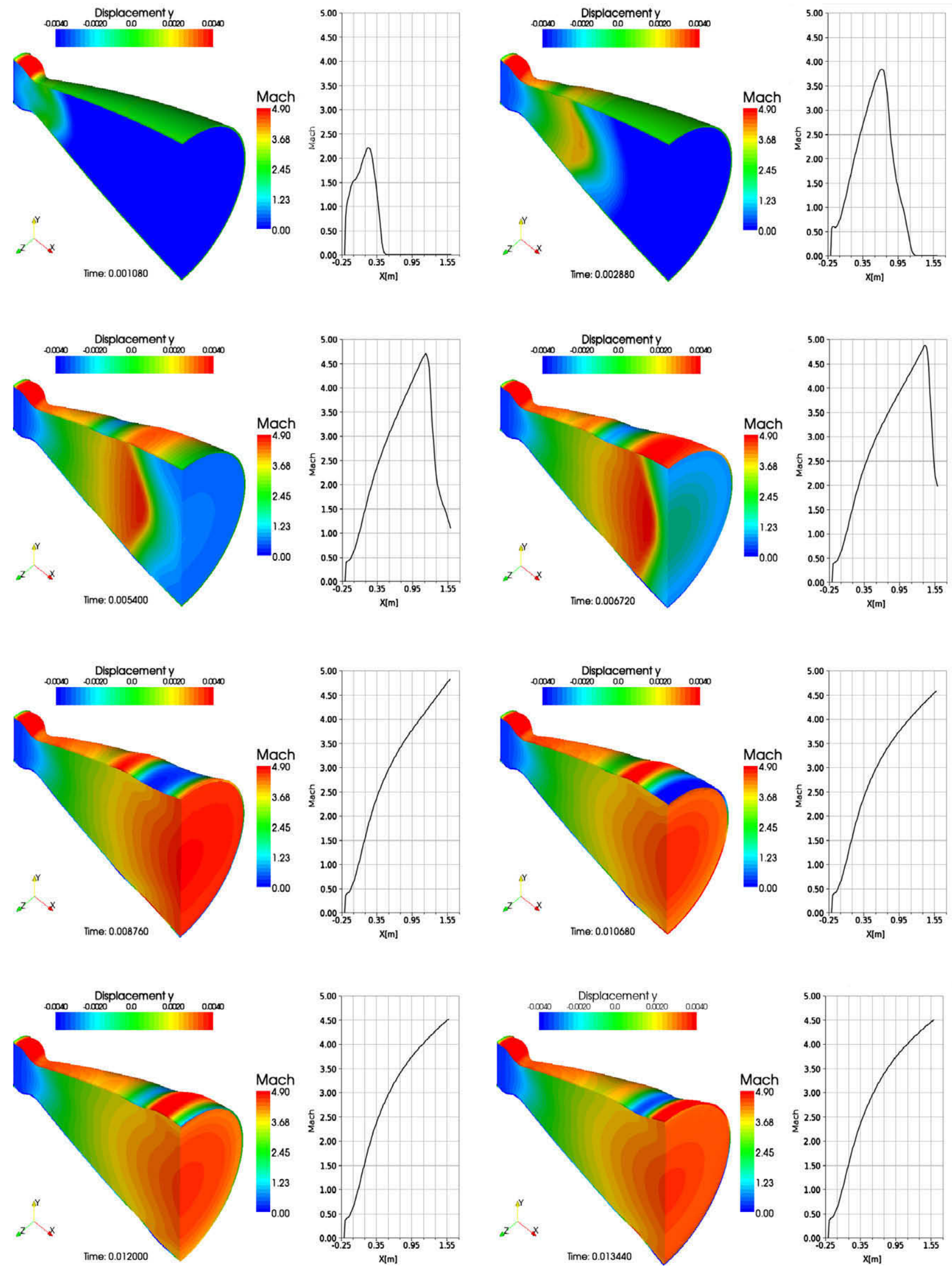

Fig. 12. Ignition process of the rocket engine. 
Therefore to perform an aeroelastic study of the proposed TOP (Thrust Optimized Parabolic or Parabolic Bell Nozzle) nozzle the radius and the wall pressure distribution $\left(p_{w}\right)$ must be comparable to the Vulcain, S1 and S3 (see Fig. 7). Fig. 7 shows that the TOP nozzle under study has similar radius distribution than Vulcain, S1 and $\mathrm{S} 3$, which makes valid the comparison between the parietal pressures. Then, the computed wall pressure when the flow is completely developed is compared, showing a good agreement.

Having verified the pressure distribution when the flow is completely developed, the next step is to study qualitatively the evolution of the shock wave during the start-up. In Ref. [21] the behavior of the structure when a shock wave moves through the divergent zone of the nozzle is described and the process is outlined in Fig. 8.

During the start-up process the pressure increases linearly from $p_{\infty}$ to $p_{0}$ in $1 \times 10^{-4}$ seconds. The formed shock moves rapidly (faster than speed of sound on the non-perturbed condition) trough the stagnant low pressure medium. Also a secondary left running (with respect to the fluid) shock wave appears and is carried to the right because of the supersonic carrier flow. This shock wave links the high Mach number, low pressure flow, with the lower velocity high pressure gas behind the primary shock. The results of the fluid structure interaction during this stage are shown in Fig. 9, together with the pressure at the wall. Note a large pressure jump across the secondary shock wave (see Fig. 9), which produces significant bending moments in the structure, changing the outflow pattern and the pressure downstream while the shock wave propagates towards outlet, making this process totally dynamic. First of all, a run is performed only considering the fluid problem (hereafter case name NO-FSI) such that the parietal pressure is computed without the effect of the wall movement. Then, the coupling is performed (case name FSI) and the parietal pressures of both cases are compared. The temporal evolution of the pressure at the nodes (1-5) located at the positions shown in Fig. 9 are plotted in Fig. 10. As seen in the Figures, the wall displacements (shown in Fig. 11) produce oscillations in the fluid pressure which are not considered for the first case (NO-FSI). As the plot shows, considering the wall displacement to compute the pressure acting in the nozzle is very important and this is one of the key points of this work.

In this case the shock wave is expelled from the nozzle but in certain operating condition, like overexpanded mode, the shock wave do not leave the nozzle. This kind of shock produce a strong pressure jump and with the structure deformation can cause an asymmetric pressure distribution as is mentioned in Östlund [4]. So, this is a first step in order to demonstrate the relation between the aeroelastic coupling and the acting lateral loads.

The sequence in Fig. 12 shows the behavior of the structure as the shock wave moves through the divergent section of the nozzle. Also, the Mach number on the nozzle centerline is plotted in the right side.

\subsection{Aeroelastic frequency shifting}

During the design of a nozzle it is important to predict the response of the structure under thrust loads, like lateral ones, because in normal operating conditions, the nozzle is subject to external and internal flows that change the wall pressure distribution dynamically. There exist several approaches to perform this analysis, starting from the simplest one, where the nozzle is characterized only by the mass, the inertia and a torsional spring at the throat, to more complex FSI models [34] as the one studied in this work.

The following analysis gives some physical insight in how the fluid forces shift the eigenfrequencies of the system due to the coupling phenomena.

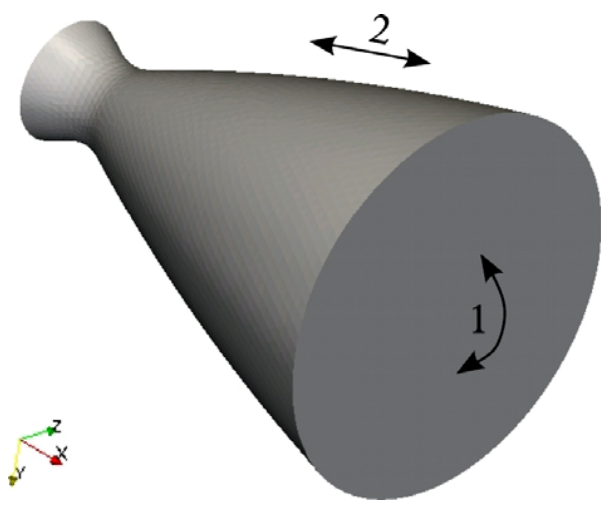

Fig. 13. Modes of vibration.

The most studied mode in the nozzle structure problem, is the lowest frequency bending mode (1) (see Fig. 13). In this work, the study is extended to the lowest frequency axial mode (2). The bending mode is excited by side loads while the axial mode is excited by fluctuations in the thrust, as occurs during start-up.

For the computation of the eigenfrequencies $\omega_{i}$ of the structure two methods are considered. One is the "hammer test" where the nozzle is deformed and then is free to vibrate, characterizing the modes and frequency via a FFT (Fast Fourier Transform). The other method is the "Generalized Eigenvalue Problem" (GEPV) in which the mass and rigidity matrix are needed to solve the system

$\left(\mathbf{K}-\omega_{i}^{2} \mathbf{M}\right) \mathbf{u}=0$.

The eigenfrequencies obtained with these methods are listed in Table 5. The next step is to compute the eigenfrequencies for the coupled problem. Therefore to obtain these eigenfrequencies, the structure and the fluid are started from a fully-developed steady flow condition computed previously, being the structure deformed with the eigenvector obtained from the GEVP corresponding to the studied modes. Then, a FFT is performed over the temporal displacement of the nodes $1-5$. The frequencies resulting after carrying out these numerical simulations are compared in the Fig. 14 This analysis shows that the influence of the fluid-structure coupling may be very important, producing a frequency shift of $47.7 \%$ for the bending mode and $8.7 \%$ for the axial mode. In addition, the frequency of the axial mode increases, while the frequency of the bending mode decreases. It will be shown with a simple analysis that this change in behavior can be explained by the sign of the additional stiffness when considering the coupling with the fluid. The governing equations for the structure are

$\mathbf{M u ̈}+\mathbf{C u}+\mathbf{K u}=F_{\text {aero }}(\mathbf{u}, \dot{\mathbf{u}}, \ddot{\mathbf{u}}, \ldots)$,

where $\mathbf{M}$ is the mass matrix, $\mathbf{C}$ is the damping matrix, $\mathbf{K}$ is the stiffness matrix and $F_{\text {aero }}$ is the aerodynamic forcing term which can be expanded in term of a series in $\mathbf{u}$ and its derivatives, from which the terms up to second order are retained.

$F_{\text {aero }}(\mathbf{u}, \dot{\mathbf{u}}, \ddot{\mathbf{u}}) \approx-\left(\mathbf{K}_{\mathrm{aero}} \mathbf{u}+\mathbf{C}_{\mathrm{aero}} \dot{\mathbf{u}}+\mathbf{M}_{\mathrm{aero}} \ddot{\mathbf{u}}\right)$,

where $\mathbf{M}_{\mathrm{aero}}, \mathbf{C}_{\mathrm{aero}}$, and $\mathbf{K}_{\mathrm{aero}}$ are the mass, damping and stiffness added by the fluid. The ratio between the fluid and the structure masses is $1 / 400$, so the mass added by the fluid is negligible and

Table 5

Eigenfrequencies.

\begin{tabular}{llll}
\hline- & FFT $(\mathrm{Hz})$ & GEVP $(\mathrm{Hz})$ & Difference $(\%)$ \\
\hline Mode 1 & 17.2 & 15.7 & 9.5 \\
Mode 2 & 138.5 & 129.9 & 6.6 \\
\hline
\end{tabular}




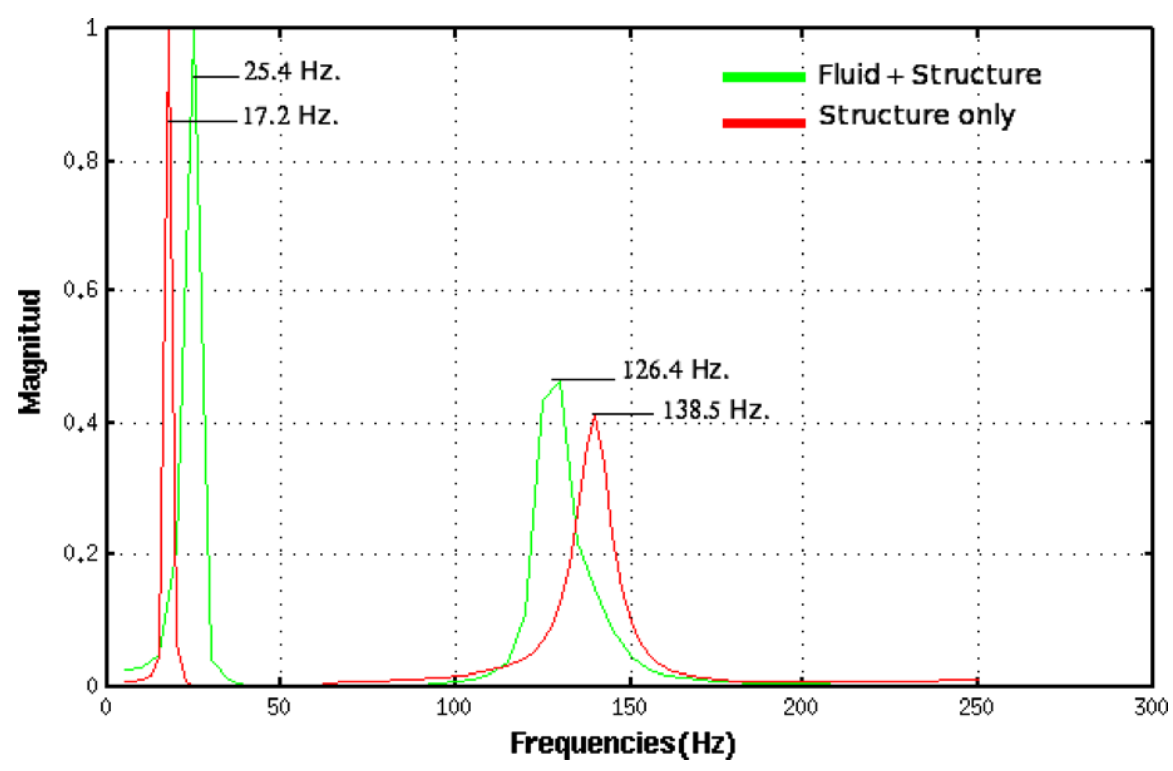

Fig. 14. Spectrum of the two studied modes.

is not taken into account in the forcing term. In order to justify this assumption, a simple test case with the fluid at rest was carried out and showed that nozzle eigenfrequencies remain unchanged compared to the case without coupling. In addition, the added mass term always tends to reduce the frequency, so that it has no effect on the shift directions. The damping term has been neglected, being its influence on frequency of second-order. So, combining the Eqs. (16) and (17), results in

$$
\mathbf{M u ̈}+\left(\mathbf{K}+\mathbf{K}_{\text {aero }}\right) \mathbf{u}=0 .
$$

We insist that the scope of these crude approximations is merely in order to have a very simple explanation for the different sign in the frequency shifts. An increment in the global stiffness gives a higher eigenfrequency and viceversa. According to the perturbation theory, the eigenfrequency shift is given by:

$\delta \omega_{i}^{2}=\frac{\psi_{i}^{T} \mathbf{K}_{\mathrm{aero}} \psi_{i}}{\psi_{i}^{T} \mathbf{M} \psi_{i}}$,

where $\delta \omega_{i}^{2}$ and $\psi_{i}$ are the change in the square of the eigenvalue and the normalized vector of displacements for the mode $i$. As the denominator is always positive, only the sign of the numerator has to be determined. It can be shown that the numerator is the work $\mathscr{W}$ done by the fluid on the structure as it vibrates in the corresponding mode,

$\psi_{i}^{T} \mathbf{K}_{\text {aero }} \psi_{i}=-\int_{S}\left(p_{i}-p_{r e f}\right)\left(\hat{\mathbf{n}} \psi_{i}\right) \mathrm{d} S=\mathscr{W}$,

where $S$ and $\hat{\mathbf{n}}$ are the inner surface of the nozzle and its normal pointing to the inside. The wall pressure distribution on $S$ for the unperturbed problem is $p_{\text {ref }}$ and $p_{i}$ is the pressure distribution corresponding to a small perturbation in the mode $i$.

Then, if the work done by the fluid is positive, the eigenfrequency is shifted to a higher value and conversely, if the work done is negative the eigenfrequency is shifted to a lower value.

Using the Eq. (20), the work done by the fluid is 1.3 (Joules) for the bending mode and it is -53.1 (Joule) for the axial mode, which explains the sign of the frequency shifts shown in Fig. 14.

\section{Conclusions}

The aeroelastic process developed during the starting phase of the rocket engine was analyzed via a coupling fluid/structure code with a weak coupling algorithm over the fluid and structure states. The code was previously validated with the study of flutter of a flat solid plate aligned with a supersonic flow, where the critical Mach number was computed and compared with the one obtained from Houbolt's approximation.

A comparative analysis of the parietal pressure of multiple TOP nozzle was carried out (Section 5.1) with the aim of validating the internal fluid flow model. The accuracy when computing the wall pressure distribution is very important because it is used in the aeroelasticity analysis, having a direct impact in computed eigenfrequencies of the coupled problem.

A modal analysis of the structure was performed via two different methods (FFT, GEPV) to obtain the eigenfrequencies of characteristic modes of the nozzle. The behavior of these modes was studied in the coupled case that represents the normal operation condition. It was verified that the effect of the coupling on the structure frequencies cannot be neglected in this case.

The use of dynamic/absorbing boundary conditions reduced significantly the CPU time and the consumed memory by allowing to put the artificial exterior boundary at the exit plane of the nozzle. Furthermore, these kind of dynamic conditions automatically handle the change in the Jacobian profile when the fluid changes its regime from subsonic to supersonic during the ignition stage.

\section{Acknowledgments}

This work has received financial support from Consejo Nacional de Investigaciones Científicas y Técnicas (CONICET, Argentina, PIP 5271/05), Universidad Nacional del Litoral (Argentina, Grants CAI+D 2005-10-64, CAI+D 2009 65/335) and Agencia Nacional de Promoción Científica y Tecnológica (ANPCyT, Argentina, Grants PICT PME 209/2003, PICT-1506/2006, PICTO-23295/2004, PICT-B 2008 Dir.Rodrigo Paz, PICT 01141/2007). Extensive use of freely distributed software such as GNU/Linux OS, MPICH, PETSc, Metis, Octave, ParaView, OpenDX and many others is done in this work.

\section{References}

[1] Dowell E, Crawley E, Curtiss H, Peters D, Scanlan R, Sisto F. A modern course in aeroelasticity. 4th ed. Dordrecht: Kluwer Academic Publishers; 1995.

[2] Pekkari LO. Aeroelastic analysis of side-loads in supersonic nozzles with separated flow. In: AIAA, SAE, ASME, and ASEE, joint propulsion conference and exhibit; 1994. 
[3] Pekkari LO. Aeroelastic stability of supersonic nozzles with separated flow. In: AIAA, SAE, ASME, and ASEE, joint propulsion conference and exhibit, 29th, Monterey, CA; 1993.

[4] Östlund J. Side-load phenomena in highly overexpanded rocket nozzles. J Propul Power 2004;20(2):695-704.

[5] Östlund J. Flow processes in rocket engine nozzle whit focus on flow separation and side-loads. Licentiate Thesis, Royal Institute of Technology; 2002.

[6] Idelsohn SR, Oñate E, Del Pin F, Calvo N. Fluid-structure interaction using the particle finite element method. Comput Methods Appl Mech Eng 2006;195(17-18):2100-23.

[7] Michler C, Hulshoff SJ, van Brummelen EH, De Borst R. A monolithic approach to fluid-structure interaction. Comput Fluids 2004;33(5-6):839-48.

[8] Felippa CA, Park KC, Farhat C. Partitioned analysis of coupled mechanical systems. Comput Methods Appl Mech Eng 2001;190(24-25):3247-70.

[9] Piperno R, Farhat C. Partitioned procedures for the transient solution of coupled aeroelastic problems. Part II: energy transfer analysis and threedimensional applications. Comput Methods Appl Mech Eng 2001;190: 3147-70.

[10] Storti MA, Nigro N, Paz RR, Dalćn DL, Lopez E. PETSc-FEM a general purpose parallel, multi-physics FEM program. <http://www.cimec.or.ar/petscfem>.

[11] Storti MA, Nigro N, Paz RR, Dalcín DL. Strong coupling strategy for fluid structure interaction problems in supersonic regime via fixed point iteration. J Sound Vib 2009;30:859-77.

[12] Farhat C, Van der Zee KG, Geuzaine P. Provably second-order time-accurate loosely-coupled solution algorithms for transient nonlinear computationa aeroelasticity. Comput Methods Appl Mech Eng 2006;195(17-18):1973-2001.

[13] Johnson AA, Tezduyar TE. Mesh update strategies in parallel finite element computations of flow problems with moving boundaries. Comput Methods Appl Mech Eng 1994:11973-94.

[14] López E, Nigro N, Storti M, Toth JA. minimal element distortion strategy for computational mesh dynamics. Int J Numer Methods Eng 2007;69(9): 1898-929.

[15] Tezduyar T, Senga M. Determination of the shock-capturing parameters in SUPG formulation of compressible flows. In: Computational mechanics WCCM IV. Beijing (China): Tsinghua University Press, Springler-Verlag; 2004.

[16] Tezduyar, Tayfun E. Finite elements in fluids: stabilized formulations and moving boundaries and interfaces. Comput Fluids 2007;36(2):191-206.

[17] Prodromou P, Hillier R. Computation of unsteady nozzle flows. In: The proceedings of the 18th. ISSW, Sendai, Japan, vol. II(24); 1992. p. 1113-8.
[18] Igra O, Wang L, Falcovitz J, Amann O. Simulation of the starting flow in a wedge-like nozzle. Int J Shock Waves 1998;8:235-42.

[19] Houbolt JC. A study of several aerothermoelastic problems of aircraft structures. Mitteilung aus dem Institut fur Flugzeugstatik und Leichtbau 5. Switzerland: E.T.H. Zurich; 1958.

[20] Hron J, Turek SA. Monolithic FEM/multigrid solver for an ALE formulation of fluid-structure interaction with applications in biomechanics. Lect Notes Comput Sci Eng 2006;53:146-70.

[21] Lefracois E. Numerical validation of a stability model for a flexible over expanded rocket nozzle. Int J Numer Methods Fluids 2005;49(4):349-69.

[22] Shimizu Taro, Kodera Masatoshi, Tsuboi Nobuyuki. Internal and external flow of rocket nozzle. J Earth Simul 2008;9:19-26.

[23] Ten-See Wang. Transient three dimensional analysis of side load in liquid rocket engine nozzle. AIAA 2004;(2004):3681.

[24] Lefracois E, Dhatt G, Vandromme D. Fluid-structure interaction with application to rocket engines. Int J Numer Methods Fluids 1999;30:865-95.

[25] Verma Shashi Bhushan, Stark Ralf, Haidn Oskar. Relation between shock unsteadiness and the origin of side-loads inside a thrust optimized parabolic rocket nozzle. Aerosp Sci Technol 2006;10(6):474-83.

[26] Moríñigo J, Salvá J. Numerical study of the start-up process in an optimized rocket nozzle. Aerosp Sci Technol 2008;12(6):485-9.

[27] Rao GVR. Approximation of optimum thrust nozzle contour. ARS J 1960;30(6):561.

[28] Sutton G, Biblarz O. Rocket propulsion elements. 7th ed. New York: John Wiley and Sons; 2001.

[29] Oates G. Aerothermodynamics of gas turbine and rocket propulsion. 3rd ed. Reston: AIAA; 1997.

[30] Mattingly J, VonOhain Hans. Elements of propulsion: gas turbines and rockets. 2nd ed. Reston: AIAA; 2006.

[31] Tuner MJL. Rockets and spacecraft propulsion. 2nd ed. New York: Springer; 2006.

[32] Storti M, Nigro N, Paz RR, Dalcín DL. Dynamic boundary conditions in computational fluid dynamics. Comput Methods Appl Mech Eng 2008;197(1316):1219-32.

[33] Guruswamy GP. Development and applications of a large scale fluids structures simulation process on clusters. Comput Fluids 2007;36(3):530-9.

[34] Sathe S, Benney R, Charles R, Doucette E, Miletti J, Senga M, et al. Fluidstructure interaction modeling of complex parachute designs with the spacetime finite element techniques. Comput Fluids 2007;36(1):127-35. 